\title{
Natal Teeth in Association with Sublingual Ulceration: A Case Report
}

\author{
Renuka P Chinchalkar ${ }^{1}$, Shantanu Jain², Shavan K Yadav ${ }^{3}$, Priyanka S Lekhwani ${ }^{4}$, Nikita Gupta ${ }^{5}$, Pooja Yadav ${ }^{6}$
}

\begin{abstract}
Natal teeth are a rare sight in a newborn child's oral cavity. The presence of teeth natal teeth can cause a series of damage to the child including difficulty in feeding, ulceration on the tongue. A 31-day-old baby reported to the Department of Pediatric and Preventive Dentistry with a chief complaint of ulceration on the tongue and difficulty in breastfeeding. On further examination, a diagnosis of natal teeth was narrowed down. Due to the presence of mobility of the present teeth and ulceration on the tongue, the teeth were extracted.

Keywords: Natal teeth, Riga-Fede disease, Sublingual ulceration.

Journal of Mahatma Gandhi University of Medical Sciences \& Technology (2021): 10.5005/jp-journals-10057-0150
\end{abstract}

\section{INTRODUCTION}

The development of the child is marked by remarkable changes within the body through fertilization to early childhood. Pediatric dentists play a major role in a child's life right from infancy to early adulthood. In normal sequence eruption of the first primary teeth begins approximately at 6 months of age. In some instances, a child may be born with a tooth-like structure within the oral cavity; such teeth are known as natal teeth. In some cases, the teeth erupt within the first month of life, such teeth are known as neonatal teeth. The occurrence of natal teeth in children is three times more common than neonatal teeth.

Natal and neonatal teeth were first reported by Titus Livius in 59 BC. There are many myths and taboos believed by people regarding such teeth. Some superstitious families believed that children with such teeth are deprived of parental love and such teeth should be removed as early as possible. In countries like India, Poland, and Africa, children with the presence of such teeth were killed immediately as it was considered as a bad omen. Another belief of people from England was that the infants born with natal teeth would become famous soldiers. Also, people from France and Italy considered such children as the future conqueror of the world. Chinese tradition believed the presence of such teeth as a bad omen for girls. ${ }^{2}$

Various other terminologies were used previously to describe natal/neonatal teeth such as dentitia praecox, dens connatalis, congenital teeth, fetal teeth, infancy teeth, pre deciduous teeth, and precocious dentition. ${ }^{3}$ The incidence of natal teeth ranges from $1: 2,000$ to $1: 3,500$ live births. Most commonly found natal teeth were lower primary central incisors. The incidence of natal and neonatal teeth is $85 \%$ in mandibular incisors, $11 \%$ in maxillary incisors, $3 \%$ in mandibular canine and molars, and only $1 \%$ in the maxillary posterior region. It is essential to diagnose radiographically whether the natal teeth are primary teeth or supernumerary teeth. More than $90 \%$ of the natal and neonatal teeth are primary teeth while only $1-10 \%$ are supernumerary teeth.

Morphologically, the appearance of natal teeth is similar to primary teeth but the size is often smaller. They are conical in shape, yellowish in color, and have hypoplastic enamel and dentin with poor or absent root development. The exact etiology

\footnotetext{
${ }^{1-6}$ Department of Pedodontics and Preventive Dentistry, Mahatma Gandhi Dental College and Hospital, Jaipur, Rajasthan, India

Corresponding Author: Renuka P Chinchalkar, Department of Pedodontics and Preventive Dentistry, Mahatma Gandhi Dental College and Hospital, Jaipur, Rajasthan, India, Phone: +91 8600778359, e-mail: r1234chinchalkar@gmail.com

How to cite this article: Chinchalkar RP, Jain S, Yadav SK, et al. Natal Teeth in Association with Sublingual Ulceration: A Case Report. J Mahatma Gandhi Univ Med Sci Tech 2021;6(2):75-77.

Source of support: Nil

Conflict of interest: None
}

of the presence of such teeth remains unknown. Previously, it was considered that the neonatal teeth are cyst of the dental lamina. It was also believed that such teeth are inherited as a dominant autosomal trait. Other causative factors include infection, malnutrition, fever, hormonal stimulation, the peripheral position of the tooth germ, and hypervitaminosis. Pre-eruption of the teeth may be associated with some syndromes such as Hallermann-Streiff syndrome, Ellis-Van Creveld syndrome, steatocystoma, multiplex, congenital pachyonychia, Wiedemann-Rautenstrauch, cleft lip, and palate. ${ }^{4}$

Pre-eruption of the teeth may lead to many complications. A major problem arising from the presence of such teeth is ulceration on the ventral side of the tongue which is caused due to the sharp incisal edge of the teeth. This ulceration causes feeding difficulties which may lead to nutritional deficiencies in such children. The presence of such traumatic sublingual ulceration in an infant is known as Riga-Fede disease. ${ }^{5}$

\section{Case Description}

A 31-day-old male child reported to the Department of Pedodontics and Preventive Dentistry, Mahatma Gandhi Dental College and Hospital, Sitapura, Jaipur with the chief complaint of difficulty in breastfeeding. The patient was struggling to breastfeed since birth; the mother also gave a history of pain during breastfeeding.

History of full-term, normal vaginal delivery, and normal birth weight of two and a half kilograms were recorded. No 


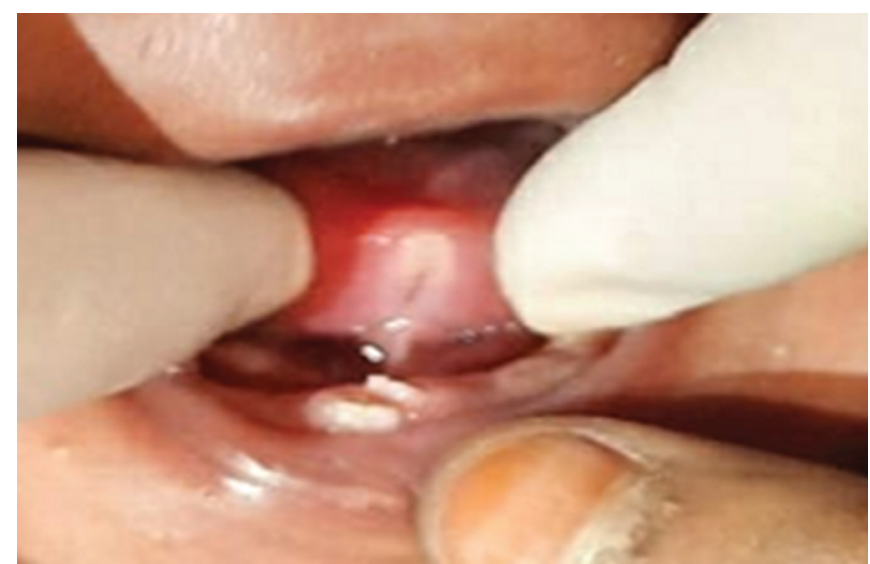

Fig. 1: Natal teeth with ulceration on the tongue

abnormality was detected in the extraoral examination. On intraoral examination, two teeth were present in the anterior region of the mandible in the area corresponding to 81 , the second tooth present was just lingual to the first tooth.

Both the teeth presented grade II mobile according to Miller's classification for the mobility of teeth. The size and shape appeared similar to normal deciduous teeth. The gum pads of the remaining mandibular arch and maxillary arch appeared to be normal.

On inspecting the ventral aspect of the tongue, there was a presence of ulceration on the tip of the tongue. The ulceration was covered with a white plaque with a hue of yellowish discoloration was also painful on palpitation. The condition was diagnosed as an ulcer progressing toward Riga-Fede disease.

Taking into account, the current scenario of COVID-19 pandemic radiographs were avoided. As the mother presented difficulty in breastfeeding and the developing ulceration on the tongue, it was decided to extract the teeth. Under local anesthesia, the two teeth were carefully extracted and the socket was compressed. The patient was advised to come for a follow-up after 15 days (Figs 1 and 2).

\section{Discussion}

The appearance of teeth at birth or within a month after birth is an infrequent condition encountered by the pediatrician or the dentist. They are classified based on the eruption of the teeth into the oral cavity. If the teeth are present immediately after birth they are considered natal teeth, while the teeth which erupt within a month of birth are classified as neonatal teeth. ${ }^{6}$ These teeth are further classified based on the appearance of the natal teeth as they emerge into the oral cavity. ${ }^{7}$ The etiology of natal and neonatal teeth is still considered questionable, though various reasons such as nutritional deficiencies, the genetic blueprint of the patient, environmental factors, superficial position of the tooth germ, infections, endocrine disturbances, and presence of any syndrome. Natal and neonatal teeth are associated with $>20$ syndromes namely Ellis-Van Creveld (chondroectodermal dysplasia), pachyonychia congenital (Jadassohn-Lewandowsky), Hallermann-Streiff (oculomandibulodyscephaly with hypotrichosis), Rubinstein-Taybi, steatocystoma multiplex, Pierre-Robin, cyclopia, Pallister-Hall, etc. ${ }^{8}$ In our present case, the child did not have any associated syndrome the present two natal teeth were prematurely erupted primary teeth, but this diagnosis can only be confirmed after 1 year with the radiographic examination.

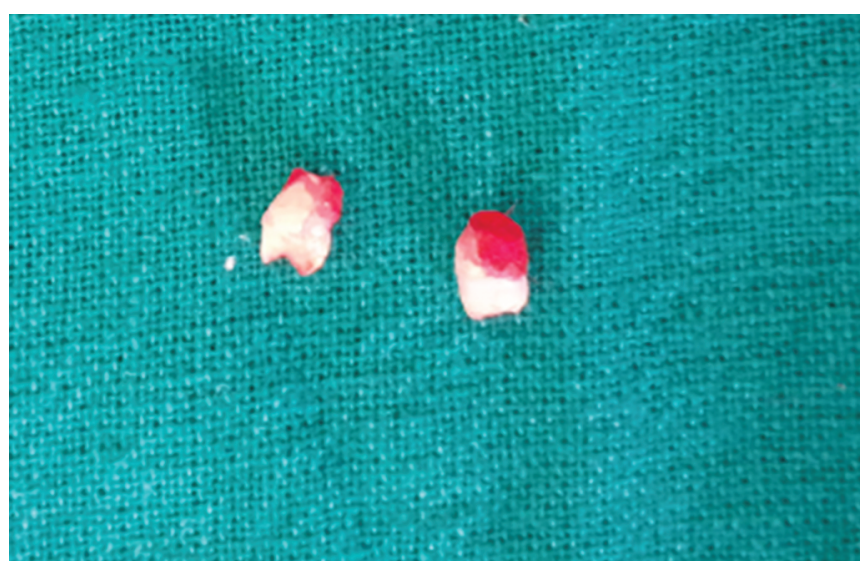

Fig. 2: Extracted natal teeth

Anatomically, natal teeth might resemble the deciduous teeth but in various instances, they are poorly developed, small, conical yellowish. They also have hypoplastic enamel and dentin, in some cases, there is the presence of roots. In the present case report, the natal teeth showed an absence of roots. ${ }^{9}$

The presence of natal or neonatal teeth can lead to various problems for the child and the mother such as difficulty in suckling, traumatic ulceration on the tongue, ulceration to the mother's nipple, and accidental aspiration during breastfeeding. ${ }^{10}$

The clinical characteristics of Riga-Fede disease are so prototypical that proper history and clinical examination are enough for diagnosis and rarely do need any histopathological investigations.

Riga-Fede disease was first mentioned by Antonio Riga in 1881. It is also called traumatic lingual ulceration, eosinophilic granuloma, and traumatic eosinophilic ulceration of the tongue and oral mucosa, sublingual fibrogranuloma, sublingual growth in infants, traumatic atrophic glossitis. It is characterized by a lesion on the tongue mucosa which occurs due to trauma by primary teeth. It can occur on any surface of the oral mucosa but the most commonly affected site is the ventral region of the tongue. ${ }^{11}$

It starts as a small ulcer with raised edges and with continuous trauma it becomes a large fibrous mass. Histopathologically, ulcerated mucosa with granulation tissue along with mixed inflammatory infiltrate of lymphocytes, mast cells, macrophages, and eosinophils are observed. This lesion causes difficulties for the child to suck and feed, thereby increasing the risk of nutritional deficiencies. It is important to identify the patients with such diseases so that proper assessment, diagnosis, and management can be performed. ${ }^{12}$

The management of Riga-Fede disease, natal, and neonatal is always a topic for debate. Many argue that the first approach for managing the patient with Riga-Fede disease should always be conservative. Tooth modification by trimming the sharp edges or by covering the sharp edges with adhesive restorations or mouthguards can be done. However, if the conservation approach does not resolve the problem, tooth extraction is indicated. ${ }^{11-13}$

In the present case, the patient had difficulty in breastfeeding and the mother experienced discomfort while breastfeeding. The two natal teeth were grade II mobile and causing ulceration on the tongue and hence the treatment decided was to extract the two teeth. After extraction of the teeth, the patient was asked to come for follow-up after 15 days. 


\section{Conclusion}

Early diagnosis of Riga-Fede disease and management of natal teeth can reduce the discomfort caused to the patient. In our case, we chose to extract the mobile natal teeth due to the threat of aspiration of these teeth by the patient. After extraction and healing of the ulceration, we confirmed that the patient was able to feed properly without causing any discomfort to the mother. Along with the treatment of the causative agent, parent counseling in such cases is extremely important.

\section{References}

1. Jose SC, Abraham KK, Khosla E. Traumatic sublingual ulceration in a newborn. J Res Dentis 2020;8(3):32-35. DOI: 10.19177/jrd.v8e320203235.

2. Dolas A, Deshpande N, Bahetwar T, et al. Natal teeth: an overview and case reports. Med J DY Patil Vidyapeeth 2019;12(1):72-74. DOI: 10.4103/mjdrdypu.mjdrdypu_80_18.

3. Bimal Deep S, Ranadheer E, Rohan B. Riga-Fede disease: report of a case with literature review. J Acad Adv Dent Res 2011;2(2):27-30.

4. Gunasekaran G, Mani G, Rengalakshmi S. Prevalence of natal or neonatal teeth in paediatric dental patients in Chennai-a retrospective study. Palarch's J Archaeol Egypt/Egyptol 2020;17(7):217-226.
5. Daraz H, Brij D, Shabir B, et al. Natal teeth, neonatal teeth and RigaFede syndrome: a case report. Ejmpr 2019;6(11):420-423.

6. Volpato LER, Simões CAD, Simões F. Riga Fede disease associated with natal teeth: two different appraoches in same case. Hindawi. 2015;2015:234961.

7. Rao RS, Mathad SV. Natal teeth: case report and review of literature. J Oral Maxillofac Pathol 2009;13(1):41-46. DOI: 10.4103/0973029X.44574.

8. Mhaske S, Yuwanati MB, Mhaske A, et al. Natal and neonatal teeth: an overview of the literature. ISRN Pediatr 2013;2013:956269. DOI: 10.1155/2013/956269.

9. Meena D, Mishra A, Singh C. Natal teeth with subsequent bilateral fusion of primary mandibular incisors - a case report. J Adv Med Dent Scie 2013;1(2):125-127.

10. Jamani NA, Ardini YD, Harun NA. Neonatal tooth with Riga-Fide disease affecting breastfeeding: a case report. Int Breastfeed J 2018;13(1):35. DOI: 10.1186/s13006-018-0176-7.

11. van der Meij EH, de Vries TW, Eggink HF, et al. Traumatic lingual ulceration in a newborn: Riga-Fede disease. Italian J Pediat 2012;38(1):20. DOI: 10.1186/1824-7288-38-20.

12. Slayton RL. Treatment alternatives for sublingual traumatic ulceration (Riga-Fede disease). Pediat Dentis 2000;22(5):413-414.

13. Cunha RF, Boer FA, Torriani DD, et al. Natal and neonatal teeth: review of the literature. Pediatr Dent 2001;23(2):158-162. 